\title{
The pursuit of knowledge
}

JD Polk

Deputy Chief Medical Officer and Chief of Space Medicine, National Aeronautics and Space Administration, Johnson Space Center, Houston, Texas, USA

KEYWORDS Chile, knowledge, mine, spaceflight, technology

DECLARATION OF INTERESTS No conflict of interests declared.

\author{
Correspondence to JD Polk, \\ Johnson Space Center, Houston, \\ TX 77058, USA \\ tel. + I 28 I 4836063 \\ e-mail james.d.polk@nasa.gov
}

As the Deputy Chief Medical Officer and Chief of the Division of Space Medicine for the National Aeronautics and Space Administration's (NASA) human spaceflight programme, I am a witness to human physiology and the ability of the human body to adapt in environments that most physicians do not get to see.

I am often asked if the lessons from spaceflight translate to the ground, and if the pursuit of that knowledge in the heavens above is worth the cost. Is the orbiting laboratory of the International Space Station yielding data that will be useful to future generations? What is the value of what we learn?

The pursuit of knowledge has a cascade that none of us can foresee. History has told us that the final destination is of less importance than the journey itself. It is doubtful that any explorer could have foreseen the cascade and evolution of their journey or discovery, whether that exploration was in the field or in the lab. But bold exploration almost always yields unexpected fruit.

In the past two years alone the orbiting platform of the International Space Station has yielded many clues to human physiology and medical treatment. We have begun to see astronauts with papilloedema, and followup magnetic resonance scanning has shown this to be secondary to optic nerve oedema from elevated intracranial pressure. Thus the cephalad fluid shift and prolonged enclosed environment of spaceflight have opened up clues to the world of idiopathic intracranial hypertension. ${ }^{1,2}$ Treatments and modalities developed for astronauts may well have implications for those who suffer with the ground analogue in the very near future. Likewise, the non-invasive devices developed for spaceflight measurement of intracranial pressure may have implications for the measurement of traumatic brain injury patients in the field or emergency department.

We have noted that bone density is but half the story in regards to fracture risk, and that microarchitecture of bone is of equal importance..$^{3-6}$ Whereas density may recover with the use of certain medications or rehabilitation, the microarchitecture lags behind such that the bone approximates its preflight density within three years, but it does not return to its preflight trabecular architecture. NASA has been slow to use bisphosphonates for this reason, as it appears that density represents but a slice of the fracture risk. It offers clues to the debate on bisphosphonates in the younger population with osteopenia, and why bisphosphonates may cause overmineralisation that may place certain patients at risk for lateral stress fractures. We have noted which regimens and dosages of Vitamin D3 are optimal, and which have less than ideal results. ${ }^{7}$

Advances in telemedicine allow us to perform remotely guided ultrasound and acquire real-time images of the back of the retina from a vehicle moving at 28,163 km per hour at $340 \mathrm{~km}$ above the Earth. ${ }^{8}$ These are but small slices of the knowledge gained in the orbiting laboratory of the International Space Station.

But not even NASA can foresee where the knowledge gained will cascade or provide insight in the future. There is no better example of this than the recent Chilean mine accident. The crisis in the Chilean mine vividly showcases where the pursuit of knowledge in one venture yields fruit in another. Thirty-three miners lay trapped $730 \mathrm{~m}$ below the Earth's surface. Because their situation was unprecedented, the Chilean health authorities searched and scoured the entire globe looking for answers that would help the men to survive underground for months, as well as get to the surface intact. NASA happened to be one of the agencies they asked for help.

The NASA recommendations ranged from the initial diet plan to avoid 'refeeding syndrome' in starving patients to psychological support of groups of people in isolated environments and the requirements for the rescue escape pod that would carry the men to the surface. Each of these were based on lessons learned from spacecraft contingency planning, support of longduration space crews, and human factors in vehicle design. A fluid-loading protocol and pressure garments to avoid orthostatic hypotension whlile the men were standing upright in the escape capsule were also derived from the Space Shuttle and Russian Soyuz protocols for the avoidance of orthostasis on re-entry. 
Certainly in its wildest dreams NASA would not have thought the lessons learned from human spaceflight would have had such immediate applicability to the mining industry. But challenges and extraordinary circumstances caused the Chileans to search for the answers they needed in the four corners of the Earth, and even above it. The Chileans exemplified the bold pursuit of knowledge and showed uncanny wisdom and innovation in how they acquired the answers they needed in order to execute a successful rescue. Although many of NASA's recommendations were adopted, the real credit goes to the Chilean government and health authorities for the exemplary execution of a seemingly flawless rescue operation.
From the medical perspective, we must continue to search for answers and insight into the human condition, the human response and the fight of disease. The endeavours of mankind in space have given us a unique laboratory in which to do this. It will continue to yield many unexpected fruits that we cannot foresee. As physicians we understand that the pursuit of knowledge is worth the cost, and that it pays many dividends in the future.

It does not matter where we go as long as we go and go boldly, whether that be in outer space or in the mines of Chile.

\section{REFERENCES}

I Lakin WD, Stevens SA, Penar PL. Modeling intracranial pressures in microgravity: the influence of the blood-brain barrier. Aviat Space Environ Med 2007; 78:932-6. doi:I0.3357/ASEM.2060.2007

2 Stocchetti N. Could intracranial pressure in traumatic brain injury be measured or predicted non-invasively? Almost. Intensive Care Med 2007; 33:I682-3. doi:I0.1007/s00I34-007-0798-5

3 Lang T, LeBlanc A, Evans $\mathrm{H}$ et al. Cortical and trabecular bone loss from the spine and hip in long-duration spaceflight. J Bone Miner Res 2004; 19:1006-12. doi:I0.1359/JBMR.040307

4 Keyak JH, Koyama AK, LeBlanc A et al. Reduction in proximal femoral strength due to long-duration spaceflight. Bone 2009; 44:449-53.

5 Lenart BA, Neviaser AS, Lyman S et al. Association of low-energy femoral fractures with prolonged bisphosphonate use: a case control study. Osteoporos Int 2009; 20:1353-62. doi:10.1007/ s00198-008-0805-x

6 Lenart BA, Lorich DG, Lane JM. Atypical fractures of the femoral diaphysis in postmenopausal women taking alendronate. $N$ Engl J Med 2008; 358: I 304-6. doi: I0.1056/NEJMc0707493

7 Smith SM, Gardner KK, Locke J et al.Vitamin D supplementation during Antarctic winter. Am J Clin Nutr 2009; 89:1092-8. doi:I0.3945/ajen.2008.27I89

8 Dulchavsky SA, Sargsyan AE, Garcia KM et al. Intuitive ultrasonography for autonomous medical care in limited-resource environments. Acta Astronautica 2009; epub 25 Sept 2009. doi:I0.1016/j.actaastro.2009.08.024

\section{SENIOR FELLOWS' CLUB PRIZE}

The Journal of the Royal College of Physicians of Edinburgh is proud to announce the launch of a new prize, made available through the generosity of the Senior Fellows' Club.

This prize of $£ 200$ will be awarded to the first-named (or corresponding) author of an original research paper on a clinical topic, deemed by a panel of judges to be the best paper by a doctor-in-training (i.e. pre-consultant level) published in the JRCPE in $201 \mathrm{I}$.

Further details may be obtained from the Editorial Office, RCPE, 9 Queen Street, Edinburgh, EH2 IJQ, tel 013। 2473652 or email editorial@rcpe.ac.uk. 